\title{
SPINDLES, SPHERES AND A FEW JETS: THE RADIATION GASDYNAMICS OF PLANETARY NEBULAE
}

\author{
ADAM FRANK \\ Astronomy Department, FM-20, University of Washington, Seattle WA 98195, U.S.A.
}

\begin{abstract}
We present the results of accurate numerical radiation-gasdynamic models for spherical (1-d) and aspherical (2-d) planetary nebulae (PNe). The models confirm and surpass previous efforts by recovering the detailed morphologies, kinematics, and ionization structure of real PNe. Our 1-d simulations include the evolution of the central star and a superwind/AGB wind interaction. The importance of the forward wind-blown shock and time-dependant ionization in redistributing mass in the nebulae is demonstrated. Our 2-d simulations, the first of their kind, confirm that aspherical PNe can develop through the interaction of a spherical fast wind with an aspherical superwind. We demonstrate that the full range of nebular morphologies can be recovered by considering the combination of wind/wind interactions, the ionization state of the gas and the projection angle on the sky
\end{abstract}

\section{Introduction}

The last decade has seen a number of 1 and 2-d interacting wind PNe simulations of various degrees of sophistication. These models have been successful in demonstrating the the role of interacting stellar winds in the formation of PNe (see Marten and Schoenberner (1991) and Soker and Livio (1989) for the best examples of the work done so far). Previously published models have, however, suffered from a less than accurate treatment of the radiation or the gasdynamics or both. In an effort to produce models which can be compared, in detail, with observations of the morphology, kinematics, and ionization structure of real PNe we have constructed a new radiation-gasdynamic code which simulates the evolution of interacting wind PNe. Our code uses an accurate gasdynamic numerical method. We can simulate the interaction of the winds without appeal to artificial boundary conditions and resolve all the important nonlinear waves. In addition we have included the majority of important time-dependant radiation transfer and microphysical processes in a self-consistent way allowing us to model the details of the nebular ionization, heating and cooling. A natural consequence of this treatment is the production of detailed numerical "observations" of the model for comparison with what is seen on the sky.

\section{Spherically symmetric models}

Our models begin with a '3-wind' interaction similar to that used by Marteen and Schoenberner (1991). A fast wind projects into a superwind which is already expanding 

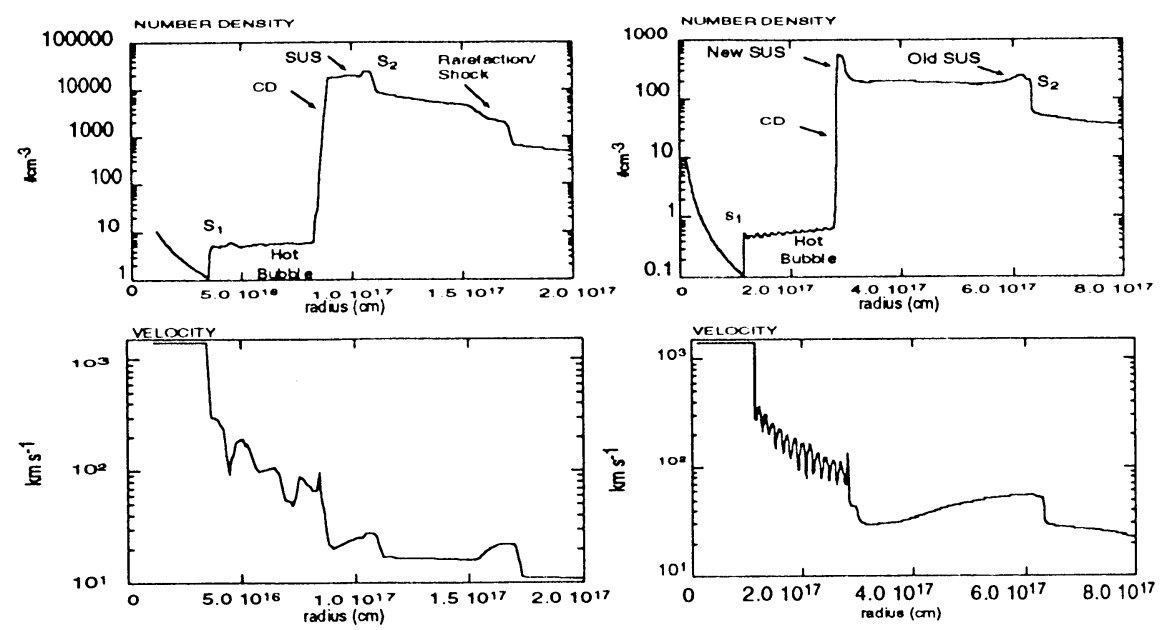

Fig 1. Density and Velocity during two phases of model PNe evolution. The frames on the left are taken from the Hydrogen Ionization Phase. The frames on the right are taken from the Shock Break-Out Phase.

into an AGB wind. The interaction of the winds occurs in the presence of a central star evolving along a $.644 \mathrm{M}$ Schoenberner track.

We find the evolution of the model is determined by the ionization state of the gas and the evolution of the principle gasdynamic waves. Fig 1 . shows the density and velocity in what we have called the Hydrogen Ionization (HI) and Shock Break-Out (SBO) evolutionary phases (in general the model evolution can be broken up into 4 distinct phases, Frank (1992)). Examination of the density in the HI phase demonstrates the codes' ability to resolve the classical wind interaction features: an inner shock $\left(S_{1}\right)$, hot bubble, contact discontinuity (CD), swept-up shell (SUS), and an outer or forward shock $\left(S_{2}\right)$. We also observe a rarefaction and shock wave occurring at the superwind/AGB wind boundary driven by an isothermal (due to ionization) pressure gradient. The velocity shows these same features as well as numerical noise in the hot bubble region. Eventually the forward shock will reach and accelerate through the superwind/AGB wind boundary. When this occurs the dynamics of the model changes appreciably and we enter the SBO phase. The density and pressure in the swept-up gas decrease in response to the acceleration allowing the hot bubble to drive a new shock into the superwind. A two shell morphology, clearly apparent in fig 1 , develops and is maintained until the end of the simulations at $8000 \mathrm{y}$.

The observation of the SBO phase in model PNe is new and demonstrates the importance of resolving the energy conserving hot bubble, the isothermal forward shock and the swept up shell. The forward shock and SUS are crucial to modeling the morphological and kinematic evolution of PNe. Fig 2 shows the $\mathrm{H} \alpha$ intensity profiles for two real PNe and for the model HI and SBO phases. The model is successful in recovering the familiar double peak profile as well as the linear shells, crowns and sharp edges investigated in Frank, Balick, and Riley (1990). Synthetic kinematical images demonstrate that the model successfully 

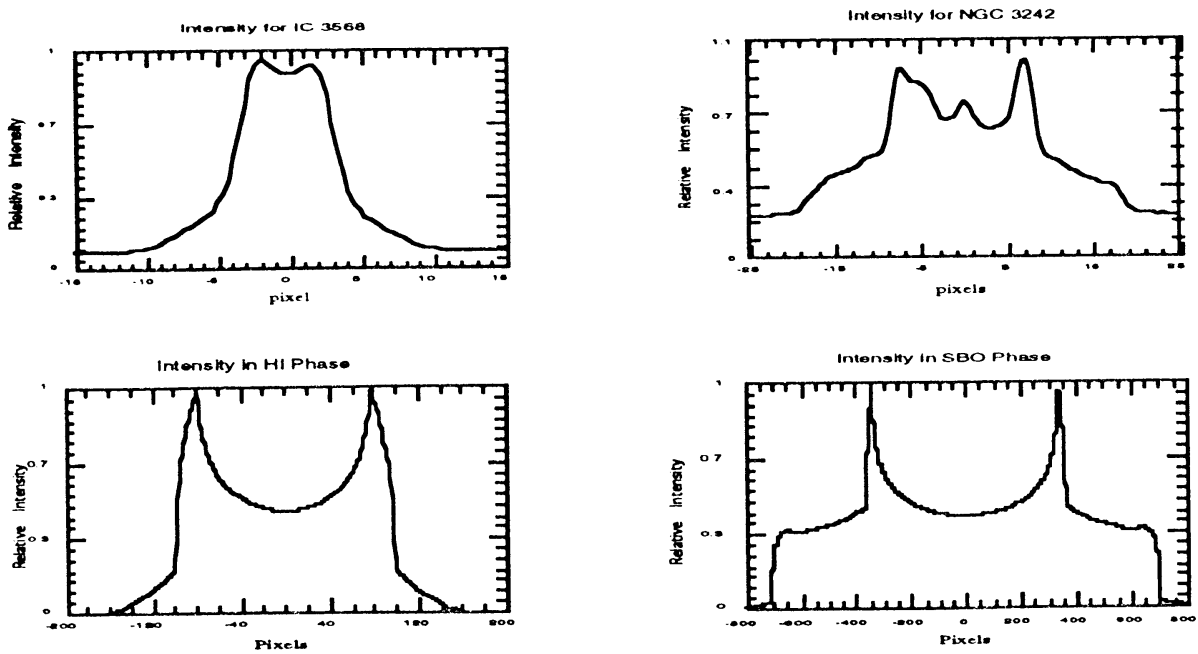

Fig 2. $\mathrm{H} \alpha$ intensity profiles for two real PNe and during two phases of model evolution. Note that the model intensity profiles have not been smoothed to account for seeing.

predicts the velocity patterns in real PNe. With the SBO phase we can embrace the full range of attached multiple shell PNe kinematical classifications enumerated by Chu et al (1989). Finally, comparison of model intensity profiles for emission lines of various ions (HeII, NII, OIII) with data show that the code is doing a good job of recreating the microphysical state of the nebular gas. In particular the models predict HeII/HeIII ionization fronts trapped in SUS for a significant fraction of the evolution of the nebulae. Many PNe show these kinds of fronts (Balick 1987).

\section{Aspherical models}

Beginning with the assumption of an aspherical superwind Mellema, Eulerink, and Icke (1991) and Icke, Balick, and Frank (1992) have performed accurate simulations of the adiabatic interaction of stellar winds in 2-d. Their results confirm that elliptical and bipolar PNe can be embraced by this generalized interacting winds scenario. They have also observed new, gasdynamical flow patterns of great potential significance to the study of PNe, these include flow focusing via an elliptical inner shock and an effective mechanism for the production of jets (Icke et al 1992).

In order to explore more realistic scenarios and to make more detailed contact with observations we have used a 2-d version of our radiation-gasdynamic code and calculated a number of PNe models (Frank 1992, Frank et al 1992). Our results demonstrate that the generalized interacting wind scenario, when combined with the effects of radiation losses and projection effects, can account for the full range of PNe morphologies. As with real PNe, the morphologies which develop in our models range from mildly elliptical, through 


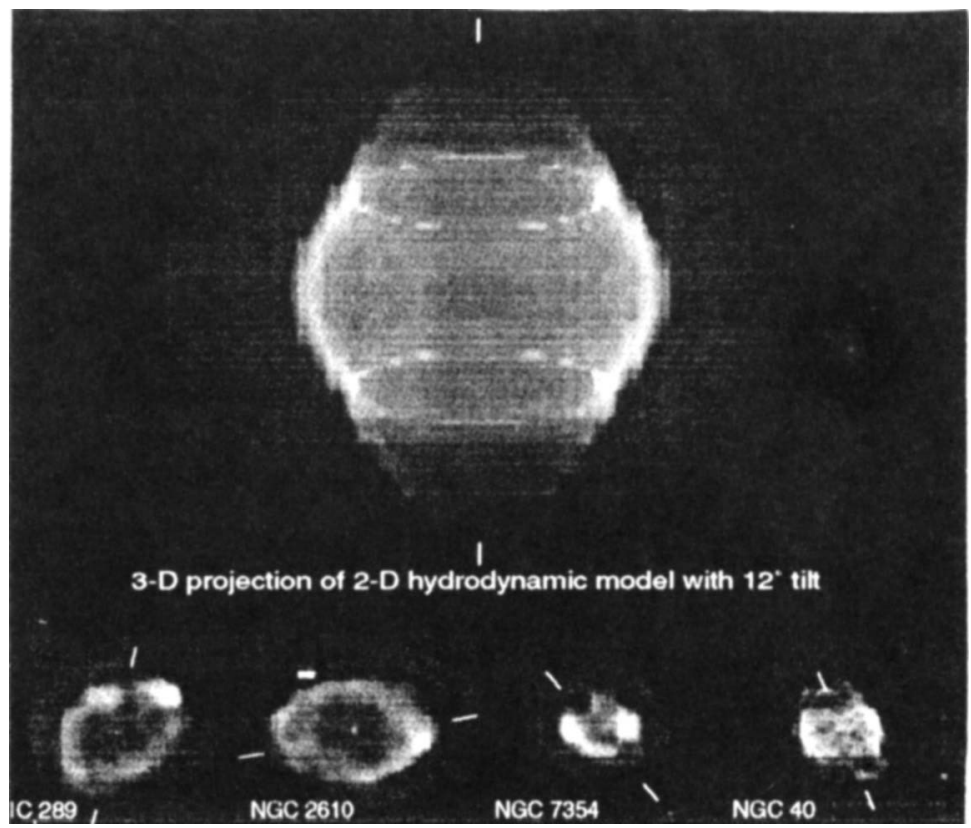

Fig 3. $\mathrm{H} \alpha$ intensity map for $2-\mathrm{d}$ model inclined inclined $12^{\circ}$ from the plane of the sky. Also shown are $\mathrm{H} \alpha$ intensity maps for $4 \mathrm{PNe}$ with similar morphologies.

spindle-like bipolars, to true peanut shaped bipolars. Fig 3 shows an $\mathrm{H} \alpha$ intensity map for a 900 year old bipolar model inclined $12^{\circ}$ from the plane of the sky. Below the model we show a number of real PNe with similar morphologies. We are in the process of calculating kinematic images of these models for more detailed comparisons with the data. The next step is to perform highly structure comparisons of the models with individual PNe.

\section{References}

Balick, B., (1987), " The Shapes and Shaping of PNe", A.J., 94, 671

Chu, Y.H., (1989), "Multiple Shell PNe", Proceedings of IAU Symposium No. 131, ed TorresPeimbert,S., (Kluwer, Dordrecht)

Frank, A., (1992), "Radiation-Gasdynamics of PNe", Thesis, University of Washington

Frank, A., Balick, B. and Riley, J., (1990), "Stellar Wind Paleontology", A.J., 100, 1903

Frank, A, Balick, B, Icke, I., and Mellema, G, (1992), "Radiation-Gasdynamics of Aspherical PNe", in preparation

Icke, I., Mellema, G., Balick, B., Eulerink, F., and Frank, A., (1992), "Collimation of Astrophysical Jets by Interial Confinement", Nature, 355

Icke, I., Balick, B., and Frank, A., (1992), "The Hydrodynamics of Aspherical PNe II",A.A., 253, 224

Mellema, G., Eulerink, F., and Icke, I.,(1992), "Hydrodynamical Models of Aspherical PNe", A.A., 252, 718

Marten, H., and Schonberner, D., (1991), "On the Dynamical Evolution of PNe", $A \& A, 248,590$

Soker, N., and Livio, N., (1989), "Interacting Winds and the Shaping of PNe", Ap.J., 339, 268 